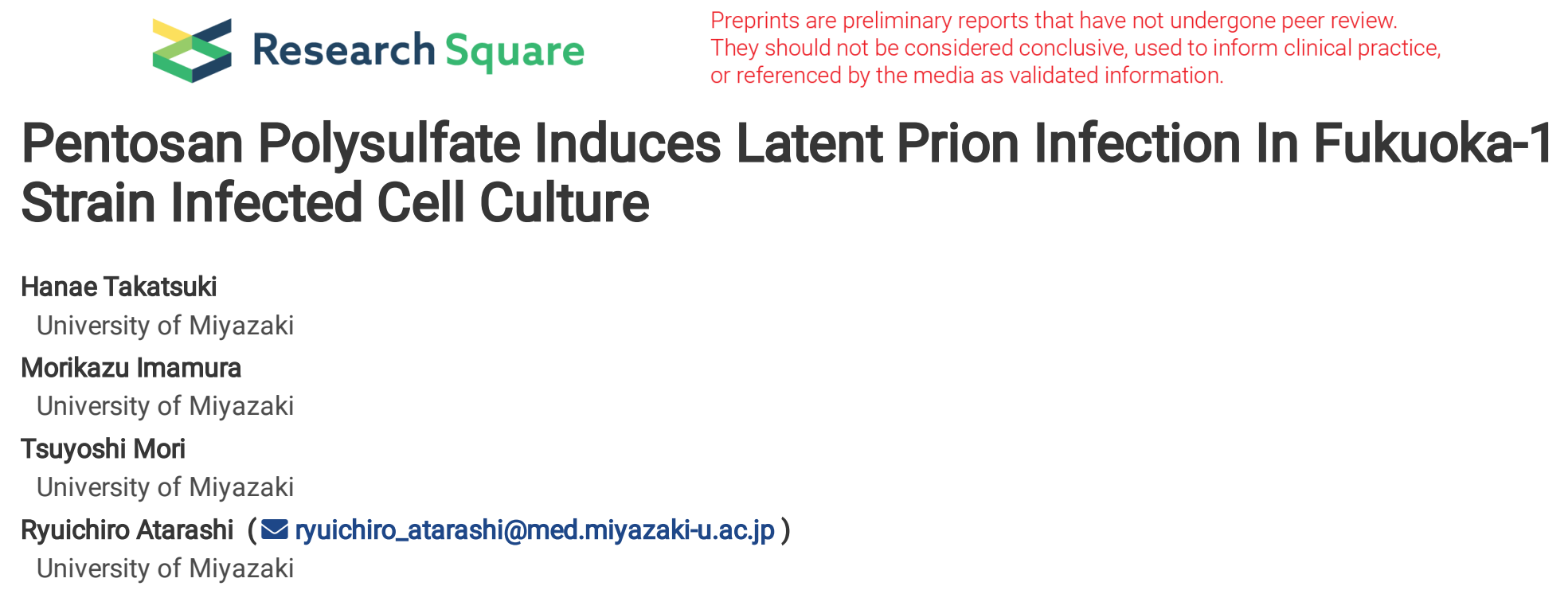

\section{Pentosan Polysulfate Induces Latent Prion Infection In Fukuoka-1 Strain Infected Cell Culture}

Research Article

Keywords:

Posted Date: March 3rd, 2022

DOl: https://doi.org/10.21203/rs.3.rs-1371679/v1

License: (c) (i) This work is licensed under a Creative Commons Attribution 4.0 International License. Read Full License 


\section{Abstract}

Each prion strain has its own characteristics and the efficacy of anti-prion drugs varies. Screening of prion disease therapeutics is typically evaluated by measuring amounts of protease-resistant prion protein (PrP-res). However, it remains unclear whether such measurements correlate with seeding activity, which can be evaluated by real-time quaking-induced conversion (RT-QuIC). In this study, the effects of anti-prion compounds pentosan polysulfate (PPS), Congo red, and alprenolol were measured in N2a58 cells infected with Fukuoka-1 (FK1) or 22L strain. The compounds abolished PrP-res and seeding activity, except for N2a58/FK1 treated with PPS. Interestingly, the seeding activity of N2a58/FK1 was much lower in the presence of PPS, but that was maintained thereafter; indeed, when PPS was removed, seeding activity and PrP-res gradually recovered to their original levels. These results indicate that prion latent infection is induced by PPS in a strain-dependent manner. Furthermore, for protein misfolding cyclic amplification (PMCA), the anti-prion effect of PPS decreased in FK1 compared to 22L, suggesting that the difference in effect observed between these two occurs at the level of the direct conversion. Our findings demonstrate that the advantages of RT-QuIC and PMCA can be exploited for more accurate assessment of therapeutic drug screening, reflecting strain differences.

\section{Introduction}

Prion disease is a fatal neurodegenerative disease caused by the accumulation of abnormal prion protein ( $\operatorname{PrPSc}$ ), a structurally altered form of normal prion protein $\left(\mathrm{PrP}^{\mathrm{C}}\right)$, in the central nervous system. Human prion diseases include Creutzfeldt-Jakob disease (CJD), Gerstmann-Strässler-Schenker syndrome (GSS), and fatal familial insomnia, while animal prion diseases include bovine spongiform encephalopathy (BSE) in cattle, scrapie in sheep, and chronic wasting disease in deer. Some regions of PrPSc are protease-resistant and remain after proteinase K (PK) treatment, which can be detected by western blotting (WB) as proteaseresistant PrPSc (PrP-res) ${ }^{1}$.

The main therapeutic targets of prion diseases are inhibition of PrP-res production, promotion of its degradation, and reduction of its neurotoxicity for early-stage cases, as well as regeneration of neural tissue for advanced cases. To screen for therapeutic agents for prion diseases targeting inhibition of PrP-res production and promotion of its degradation, candidate compounds are often added to cultured cells infected with scrapie strains, and the effect on PrP-res levels is examined by WB. Various anti-prion compounds have been found to reduce PrP-res levels in cultured cells and infected animals ${ }^{2,3}$. For example, pentosan polysulfate (PPS) ${ }^{4}$, Congo red ${ }^{5}$, and alprenolol ${ }^{6}$ can reportedly reduce PrP-res levels in prion-infected cultured cells. Among such drugs, PPS reduced accumulation of PrPSc in the brain and/or prolonged survival in prion-infected mice ${ }^{7-9}$. In addition, PPS has been used in clinical practice for variant CJD and other prion diseases, although the number of cases was limited ${ }^{10,11}$. To date, no effective prophylactic or therapeutic agent for prion diseases has been identified in clinical trials.

Detection of PrP-res by WB may be insufficient for use of PrP-res as an index of anti-prion effects (in terms of detection sensitivity) because prions can remain even after PrP-res disappears. Therefore, we developed a highly sensitive in vitro prion-detection method, the real-time quaking-induced conversion (RT-QuIC) assay ${ }^{12}$. RT-QuIC uses Escherichia coli-derived recombinant PrP as a substrate to promote the conversion reaction by agitation in samples suspected of containing prions (seed), and the process is measured as the fluorescence intensity of thioflavin $T$ to determine the presence of prions ${ }^{13}$. Numerous reports show the usefulness of RT-QuIC for diagnosis of prion diseases in humans and animals using cerebrospinal fluid and other specimens ${ }^{14}$. In combination with a serial dilution method, RT-QuIC can also measure the seeding activity as a $50 \%$ seeding dose $\left(\mathrm{SD}_{50}\right)^{15}$ with more than $10^{3}$-fold higher sensitivity than detection of PrP-res by WB ${ }^{16}$. Therefore, RT-QuIC may be more useful than WB for measuring anti-prion effects $^{17}$. The purpose of this study was to measure the effects of anti-prion compounds on seeding activity using the RT-QulC method and compare the results with PrP-res levels to determine whether RT-QuIC can improve screening for prion disease drugs.

\section{Results}

\section{Seeding activity of cell cultures persistently infected with prions can be measured by RT-QulC.}

To first determine the seeding activity of prion-infected cells, suspensions of N2a58 cells persistently infected with the FK1 or $22 \mathrm{~L}$ strain were used for quantification by the Endpoint RT-QuIC method (Fig. 1). RT-QulC reactions were performed in four independent wells using each diluted cell suspension as a seed. No increase in thioflavin T fluorescence was observed in uninfected N2a58 cells 
or without cell seeding (PBS only), whereas an increase in fluorescence was observed relatively quickly in all wells when more than 5 prion-infected cells were added (Fig. 1A). To clearly quantify seeding activity, the RT-QuIC reaction was performed three times and the mean value was calculated to determine the $50 \%$ seeding dose per $10^{6}$ cells $\left(\mathrm{SD}_{50} / 10^{6}\right.$ cells) (Fig. $\left.1 \mathrm{~B}\right)$. Log $\mathrm{SD}_{50} / 10^{6}$ cells values for N2a58/FK1 and N2a58/22L were $6.97 \pm 0.14$ and $6.22 \pm 0.29$, respectively (Table 1).

Table 1

Seeding activity in anti-prion compounds treated prion-infected cells

\begin{tabular}{|c|c|c|c|c|c|c|c|c|}
\hline \multirow{3}{*}{$\begin{array}{l}\text { Passage } \\
\text { No. }\end{array}$} & \multicolumn{8}{|c|}{ Log SD $50 / 10^{6}$ cell (mean \pm S.D.) } \\
\hline & \multicolumn{2}{|l|}{ untreated } & \multicolumn{2}{|l|}{ PPS } & \multicolumn{2}{|l|}{ Congo red } & \multicolumn{2}{|l|}{ Alprenolol } \\
\hline & N2a58/FK1 & N2a58/22L & N2a58/FK1 & N2a58/22L & N2a58/FK1 & N2a58/22L & N2a58/FK1 & N2a58/22L \\
\hline 0 & $6.97 \pm 0.14$ & $6.22 \pm 0.29$ & - & - & - & - & - & - \\
\hline 1 & - & - & $6.22 \pm 0.14$ & $4.63 \pm 0.14$ & $6.05 \pm 0.25$ & $5.47 \pm 0.72$ & $6.30 \pm 0.43$ & $5.38 \pm 0.29$ \\
\hline 2 & - & - & $5.47 \pm 0.14$ & $<3.38$ & $4.80 \pm 0.43$ & $4.47 \pm 0.38$ & $4.63 \pm 0.52$ & $<3.80$ \\
\hline 3 & - & - & $4.47 \pm 0.14$ & - & $<3.22$ & $<3.3$ & $<3.80$ & $<3.55$ \\
\hline 6 & - & - & $5.13 \pm 0.52$ & - & - & - & - & - \\
\hline 9 & - & - & $4.72 \pm 0.29$ & - & - & - & - & - \\
\hline
\end{tabular}

N2a58/FK1 treated with PPS exhibited persistent seeding activity.

Seeding activities of N2a58/FK1 and N2a58/22L cultured in the presence of PPS, Congo red, or alprenolol (which have reported antiprion effects) were quantified by RT-QuIC. Seeding activities of prion-infected cells treated with Congo red or alprenolol both decreased and became below the detection limit after the sixth passage (P6) (Fig. 2 and Tables 1). In the presence of PPS, the seeding activity of N2a58/22L was below the detection limit after P3, but interestingly, that of N2a58/FK1 remained at $4.72 \pm 0.29$ $\log \mathrm{SD}_{50} / 10^{6}$ cells even after P9 (Fig. 2 and Table 1). Amounts of PrP-res in PPS-treated prion infected cells were decreased in both 22L and FK1 strains, and almost disappeared at P2 and P3 (Fig. 4B). In contrast, there was little change in the amount of total PrP following PPS treatment.

In addition, we observed PrPSc signals in both N2a58/FK1 and N2a58/22L. The results showed that the signal intensity clearly decreased after $24 \mathrm{~h}$ of PPS treatment, and subsequently decreased to a level indistinguishable from that of uninfected cells after 48 h (Fig. 3).

\section{Seeding activity and PrP-res of N2a58/FK1 were restored to original levels following PPS removal.}

PPS efficiently abolished PrP-res in both N2a58/22L and N2a58/FK1. However, low levels of seeding activity (3-5 log SD $50 / 10^{6}$ cells) were maintained in N2a58/FK1 even after P28 in the presence of PPS (Fig. 4A). Therefore, we investigated whether the decrease in seeding activity elicited by PPS could be recovered by removal of PPS from the culture medium. After P4, prion-infected cells were cultured in medium without PPS. Thereafter, the seeding activity of N2a58/FK1 gradually increased such that by P25, seeding activity and PrP-res levels had returned to initial levels (Fig. 4). In contrast, seeding activity and PrP-res did not recover in N2a58/22L (Fig. 4B).

\section{Inhibitory effect of PPS on the PrP Sc conversion reaction in PMCA varied greatly among prion strains.}

We next used the PMCA method to examine whether PPS directly inhibited the PrPSc conversion reaction and whether this inhibitory effect differed among prion strains. PMCA is a method to promote the conversion reaction by fragmenting PrP multimers by intermittent sonication using $\mathrm{PrPC}^{\mathrm{C}}$ in brain homogenate as a substrate ${ }^{18}$. Compared with RT-QuIC, PMCA can amplify PrPSc without being significantly affected by solvents such as dimethyl sulfoxide. In fact, the anti-prion effect of cationic tetrapyrrole dissolved in dimethyl sulfoxide using PMCA has been reported ${ }^{19}$. PPS was added to the reaction mixture at a four-fold dilution from a final concentration of $10 \mathrm{mg} / \mathrm{ml}$ to $2.44 \mu \mathrm{g} / \mathrm{ml}$, and five prion strains (FK1, 22L, Chandler, ME7, and mBSE) were used as seeds. Inhibition 
of the PrPSc conversion reaction of PPS was strongest in ME7 (significant decrease at $2.44 \mu \mathrm{g} / \mathrm{ml}$ or higher), followed by Chandler $(39 \mu \mathrm{g} / \mathrm{ml}$ or higher) and $22 \mathrm{~L}(156 \mu \mathrm{g} / \mathrm{ml}$ or higher), but occurred in FK1 and mBSE only at $10 \mathrm{mg} / \mathrm{ml}$ (Fig. 5).

\section{Discussion}

In the present study, we found that even after PPS elicited the disappearance of PrP-res from N2a58/FK1, RT-QuIC results indicated that a measurable level of seeding activity remained despite passaging 28 times in the presence of PPS. Furthermore, this residual seeding activity and loss of PrP-res recovered to original levels after PPS removal, indicating that prions continued to grow in a latent infectious manner in the presence of PPS. Previously, latent prion infection was known to occur in prion transmission between heterologous animals, often referred to as a "carrier state" in which PrP-res accumulation and seeding activity exist in the host body, but the disease does not develop ${ }^{20}$. For example, in a United Kingdom (UK) study of 32,441 appendix samples, 16 were positive for PrP-res ${ }^{21}$. It would be interesting to see how many of these samples would be positive in terms of the definition of latent infection if examined by RT-QuIC. As a different mechanism from heterologous infection, our findings using cultured cells are, to our knowledge, the first to show that latent prion infection can be caused by drugs.

In contrast, the seeding activity of N2a58/22L was reduced to below the detection limit and did not recover after PPS removal. This result is consistent with previous reports indicating that PrP-res did not return after PPS treatment of scrapie-infected neuroblastoma cells ${ }^{4}$ or a transformed deer cell line persistently infected with chronic wasting disease followed by passaging in PPS-free medium $^{22}$.

Although PPS inhibited the PrPSc conversion reaction in PMCA, this effect varied greatly depending on the strain, with much weaker inhibitory effects observed for FK1 and mBSE strains compared with scrapie-derived mouse adapted strains ME7, Chandler, and 22L. FK1 is a mouse-adapted strain of GSS (P102L mutation), a hereditary prion disease, while mBSE is a mouse-adapted strain of classical BSE. The fact that both these strains were resistant to PPS suggests a gap in the conversion mode between them and the three scrapie-derived strains. The results of PMCA may explain why latent prion infection occurred only in N2a58/FK1. Specifically, heparin, a type of polyanion, binds to $\mathrm{PrP}^{\mathrm{C} 23}$ and promotes prion replication in $\mathrm{PMCA}^{24}$. PPS, a heparin analog, may compete with heparin or directly interact with $\operatorname{PrP}^{\mathrm{C}}$ independently of heparin ${ }^{25-28}$ to inhibit prion replication in vitro and in vivo. Accordingly, differences in the final $\mathrm{PrP}^{\mathrm{SC}}$ structure of each prion strain led to differences in the conversion process of $\operatorname{PrP}^{\mathrm{C}}$ bound to heparin and/or PPS, which may be the reason why the anti-prion effect of PPS was strain-dependent. Intraventricular infusion of PPS prolonged the survival of mice infected with RML (the alias of Chandler strain) or FK1, but prolongation of the FK1 incubation period was reportedly shorter than that of RML, demonstrating strain dependence of the anti-prion effect of PPS at the animal level ${ }^{9}$. Although the relevance to PPS is unclear, it has also been reported that, unlike Chandler and 22L, FK1-infected cultured cells exhibited significant changes in PrP-res in response to autophagy accelerators and inhibitors ${ }^{29}$, which may corroborate differences in conversion mode and $\mathrm{PrPSc}^{\mathrm{S}}$ structure.

Clinical trials of PPS for patients with prion disease have been conducted in Japan ${ }^{30}$ and the UK ${ }^{10,11}$. Although there is a report of one case in which postmortem examination showed a significantly reduced level of PrP-res in the brain compared with levels in brains without PPS treatment ${ }^{31}$, there was no obvious improvement in clinical symptoms in any case of familial, ectopic, or sporadic CJD treated with PPS in Japan ${ }^{30}$. Notably, in two clinical studies of UK patients with variant CJD, three out of four ${ }^{10}$ and four out of five patients ${ }^{11}$ survived longer than those who did not receive PPS treatment. These differences may result from variations in the efficacy of PPS depending on the subtype of CJD. At present, it remains difficult to determine whether PPS is effective for treating patients with CJD because the number of patients is small and outcome criteria are unclear.

Previous screening has typically identified anti-prion compounds targeting inhibition of PrP-res accumulation in prion-infected cell cultures $^{32}$. In this study, we demonstrated that seeding activity can remain even after PrP-res is lost in a strain-dependent manner. Therefore, the highly sensitive seeding activity measured by RT-QulC is a useful indicator for screening of prion disease therapeutics in prion-infected cultured cells, whereas measurement of PrP-res levels alone is likely insufficient. Furthermore, the finding that different prion strains exhibit varying effects to drugs is yet another factor complicating the development of therapeutic agents for prion diseases ${ }^{33-35}$. Screening of prion-infected cells is limited by the difficulty of persistently infecting cells with certain prion 
strains, especially CJD. Our results show that even for prion strains that cannot be persistently infected in cultured cells, PMCA may be able to predict an inhibitory effect on the prion strain.

In conclusion, we have shown that PPS induces a latent infectious state of prions at levels negative for PrP-res and detectable only by seeding activity in a strain-dependent manner.

\section{Methods}

\section{Cell culture}

The establishment of a mouse PrP-overexpressing neuroblastoma cell line (N2a58) and 22L-infected N2a58 cells was described previously ${ }^{36}$. FK1-infected N2a58 cells were also described separately ${ }^{37}$. Cells were cultured at $37^{\circ} \mathrm{C}$ in Dulbecco's Modified Eagle's Medium (Cat. No. 08458-16; Nacalai Tesque, Kyoto, Japan) containing 10\% fetal bovine serum and penicillin-streptomycin (Cat. No. 26253-84, Nacalai Tesque) in a $5 \% \mathrm{CO}_{2}$ incubator.

\section{Administration Of Pps, Congo Red, And Alprenolol To Prion-infected Cells}

Prion-infected N2a58 cells were plated in six-well plates and treated with $20 \mu \mathrm{g} / \mathrm{ml}$ PPS (Cartrophen Vet, Biopharm Australia, Bondi Junction, Australia), $50 \mu \mathrm{M}$ Congo red (Cat. No. 09403-02, Nacalai Tesque) or $50 \mu \mathrm{M}$ alprenolol (Skajilol, Kotobuki Pharmaceutical, Nagano, Japan). Concentrations of compounds added to cultured cells in this study referred to concentrations previously reported to reduce PrP-res in prion-infected cells ${ }^{5,6,38}$. Cells were passaged every 4 days and sampled each time for WB and RT-QuIC assays.

\section{Western Blotting Of Cell Lysates}

Cell lysates were collected in lysis buffer [0.5\% Triton X-100, 0.5\% deoxycholic acid, $150 \mathrm{mM} \mathrm{NaCl}$, and $50 \mathrm{mM}$ Tris- $\mathrm{HCl}$ (pH7.5)]. Total protein concentrations of cell lysates were quantified using a BCA Protein Assay Kit (\#23225; Pierce, Waltham, MA, USA). Cell lysate samples were digested with $10 \mu \mathrm{g} / \mathrm{ml} \mathrm{PK}$ at $37^{\circ} \mathrm{C}$ for $30 \mathrm{~min}$, subjected to sodium dodecyl sulfate polyacrylamide gel electrophoresis (SDS-PAGE) on NuPAGE 12\% BisTris gels (Cat. No. NP0343; Life Technologies Corporation, Carlsbad, CA, USA), and transferred to polyvinylidene difluoride membranes (Cat. No. IPVH00010; Merck Millipore, Burlington, MA, USA). Membranes were subsequently treated with Blocking One (Cat. No. 03953-95, Nacalai Tesque) for $1 \mathrm{~h}$ at room temperature. T2 antibody, a horseradish peroxidase-conjugated anti-PrP monoclonal antibody that recognizes mouse PrP135-14039, was diluted 5000-fold and used as the primary antibody. Chemiluminescence was detected using EzWest Lumi plus (Cat. No. WSE-7120L; ATTO, Tokyo, Japan). Images were obtained by LuminoGraph I (WSE-6100, ATTO, Tokyo, Japan), a chemiluminescence imaging aparatus.

\section{Rt-quic}

Cells that served as seeds for the conversion reaction were cultured in $20 \mathrm{mM}$ ethylenediaminetetraacetic acid (EDTA) and subjected to RT-QuIC. Numbers of cells were counted with a hemocytometer. Recombinant mouse PrP (rMoPrP 23-231) was purified as previously described ${ }^{12}$. Cells serially diluted with phosphate-buffered saline (PBS) were added to $95 \mu$ l of reaction buffer [500 mM $\mathrm{NaCl}, 25 \mathrm{mM}$ PIPES (pH7.0), $10 \mu \mathrm{M}$ thioflavin T, 1 mM EDTA, and 0.001\% SDS] in 96-well plates (Cat. No. 265301; Nunc, Rochester, NY, USA). The reaction was performed in samples alternately shaken and incubated for 30 s each using an Infinite F200 (Tecan, Männedorf, Switzerland). SD $_{50}$ values were calculated using the Spearman-Kärber method.

\section{Quantification Of The Inhibitory Effect Of Pps On Pmca}

Investigation of optimal conditions for PMCA using brain homogenates, especially sonication conditions, has been previously described $^{40}$. ICR mouse brains were homogenized at 10\% (w/v) in PMCA buffer (4 mM EDTA and 1\% Triton-X100 in PBS) using a Beads Crusher (Beads Crusher $\mu$ T-12; TAITEC, Saitama, Japan) and rotation at $4^{\circ} \mathrm{C}$ for $1.5 \mathrm{~h}$. PMCA samples were prepared by adding $5 \mu \mathrm{l}$ of serial diluted PPS solution to $45 \mu \mathrm{l}$ of $10 \%(\mathrm{w} / \mathrm{v})$ ICR mouse brain homogenate with $0.01 \%(\mathrm{w} / \mathrm{v})$ prion-infected mouse 
brain, $0.3 \mathrm{mg} / \mathrm{ml}$ heparin, and $0.05 \%$ digitonin. The mixture was loaded into 0.2-ml PCR tube eight-strips (Cat. No. SKPCR8D; Seiko, Tokyo, Japan) along with 2-mm zirconia beads (Cat. No. ZB-20; TOMY, Tokyo, Japan) and positioned on the floated plate holder of a Cup horn sonicator (Misonix-3000; Cole-Parmer, Vernon Hills, IL, USA). PMCA was performed for $16 \mathrm{~h}$, consisting of 30 min incubation at $40^{\circ} \mathrm{C}$ followed by a 20-s pulse of sonication. PMCA products were digested with $40 \mu \mathrm{g} / \mathrm{ml}$ of PK buffer and heated to $95^{\circ} \mathrm{C}$ for $10 \mathrm{~min}$ in SDS sample buffer. SDS-PAGE was performed using $15 \%$ Tris-glycine gels. Western Blotting was then performed using the method described above. CS Analyzer (ATTO) was used to measure the intensity of the detected PrP-res bands; to quantify the inhibitory effect of PPS on PMCA and to compare between different blots, the intensity of PrP-res without PPS on the same membrane was set to $100 \%$.

\section{Preparation Of Prp Seed For Pmca}

Mouse-adapted scrapie strains 22L, Chandler, ME7, as well as mouse-adapted classical BSE (mBSE) were propagated in ICR mice. The mouse-adapted GSS strain Fukuoka-1 (FK1) was kindly provided by N. Nishida (Nagasaki University, Nagasaki, Japan). Preparation of prion-infected brain homogenates was previously described ${ }^{24}$.

\section{Ethics Approval}

ICR mice (11 weeks old, female) for PMCA experiments were obtained from SLC, Japan. No animal experiments were conducted in the experiments described in this manuscript. All experimental protocols using animal tissues were approved by the Animal Care and Use Committee of University of Miyazaki (approval ID: 2019-010) and were performed in accordance with relevant guidelines and regulations.

\section{Immunofluorescence Assay}

N2a58 cells were grown on four-well chambered slide cover glass (Cat. No. 154917, Nunc) and treated with 20 mM PPS. Cells were washed with PBS and then fixed with pre-warmed 4\% paraformaldehyde in PBS containing 4\% sucrose for $10 \mathrm{~min}$. After permeabilization with $0.1 \%$ Triton-X100 for $10 \mathrm{~min}$, cells were treated with $5 \mathrm{M}$ guanidine thiocyanate for 10 min and blocked with $5 \%$ fetal bovine serum in PBS. Next, cells were incubated with anti-PrP mouse monoclonal antibody 132 (mAb132) to detect PrP ${ }^{41}$. Alexa Fluor 488-conjugated monoclonal anti-mouse IgG (Cat. No. A11017, Life Technologies Corporation) was used as the secondary antibody. Cell nuclei were counterstained with 4',6-diamidino-2-phenylindole.

\section{Statistical analysis}

For WB after PMCA, statistical significance was calculated by Dunnett's multiple comparison test using GraphPad Prism (GraphPad Software, San Diego, CA, USA).

\section{Data Availability}

The datasets used and/or analysed during the current study available from the corresponding author on reasonable request.

\section{Declarations}

\section{Acknowledgments}

We thank Hiromi Iguchi and Minako Ohno for technical assistance. We also thank Motohiro Horiuchi for providing the antibody and Takeshi Yamasaki for sharing the immunofluorescence assay workflow, and Noriyuki Nishida and Takehiro Nakagaki for providing the cell lines. We thank Edanz (https://jp.edanz.com/ac) for editing a draft of this manuscript.

\section{Author Contributions}


H.T. and R.A. conceived the experiments. H.T. performed most of the experiments. T.M., M.I., and R.A. guided the experiments. H.T. and R.A. wrote the manuscript. All authors analysed data and reviewed the manuscript.

\section{Funding}

This work was supported by MSD Life Science Foundation, Public Interest Incorporated Foundation, Takeda Science Foundation, and Japan Society for the Promotion of Science KAKENHI Grant Numbers JP21K07277 and JP18H0272. This work was also supported by research grants (20KA1003) from the Ministry of Health, Labour and Welfare of Japan.

\section{Competing interests}

The authors declare no competing interests.

\section{References}

1. Prusiner, S. B. Molecular biology of prion diseases. Science 252, 1515-1522, doi:10.1126/science.1675487 (1991).

2. Sim, V. L. Prion disease: chemotherapeutic strategies. Infect Disord Drug Targets 12, 144-160, doi:10.2174/187152612800100161 (2012).

3. Teruya, K. \& Doh-Ura, K. Insights from Therapeutic Studies for PrP Prion Disease. Cold Spring Harb Perspect Med 7, doi:10.1101/cshperspect.a024430 (2017).

4. Caughey, B. \& Raymond, G. J. Sulfated polyanion inhibition of scrapie-associated PrP accumulation in cultured cells. J Virol 67, 643-650, doi:10.1128/JVI.67.2.643-650.1993 (1993).

5. Caughey, B. \& Race, R. E. Potent inhibition of scrapie-associated PrP accumulation by congo red. J Neurochem 59, 768-771, doi:10.1111/j.1471-4159.1992.tb09437.x (1992).

6. Miyazaki, Y. et al. Identification of Alprenolol Hydrochloride as an Anti-prion Compound Using Surface Plasmon Resonance Imaging. Mol Neurobiol 56, 367-377, doi:10.1007/s12035-018-1088-7 (2019).

7. Diringer, H. \& Ehlers, B. Chemoprophylaxis of scrapie in mice. J Gen Virol 72 (Pt 2), 457-460, doi:10.1099/0022-1317-72-2-457 (1991).

8. Farquhar, C., Dickinson, A. \& Bruce, M. Prophylactic potential of pentosan polysulphate in transmissible spongiform encephalopathies. Lancet 353, 117, doi:10.1016/S0140-6736(98)05395-1 (1999).

9. Doh-ura, K. et al. Treatment of transmissible spongiform encephalopathy by intraventricular drug infusion in animal models. J Virol 78, 4999-5006, doi:10.1128/jvi.78.10.4999-5006.2004 (2004).

10. Bone, I., Belton, L., Walker, A. S. \& Darbyshire, J. Intraventricular pentosan polysulphate in human prion diseases: an observational study in the UK. Eur J Neurol 15, 458-464, doi:10.1111/j.1468-1331.2008.02108.x (2008).

11. Newman, P. K. et al. Postmortem findings in a case of variant Creutzfeldt-Jakob disease treated with intraventricular pentosan polysulfate. J Neurol Neurosurg Psychiatry 85, 921-924, doi:10.1136/jnnp-2013-305590 (2014).

12. Atarashi, R. et al. Ultrasensitive human prion detection in cerebrospinal fluid by real-time quaking-induced conversion. Nat Med 17, 175-178, doi:10.1038/nm.2294 (2011).

13. Atarashi, R., Sano, K., Satoh, K. \& Nishida, N. Real-time quaking-induced conversion: a highly sensitive assay for prion detection. Prion 5, 150-153, doi:10.4161/pri.5.3.16893 (2011).

14. Hermann, P. et al. Biomarkers and diagnostic guidelines for sporadic Creutzfeldt-Jakob disease. Lancet Neurol 20, 235-246, doi:10.1016/S1474-4422(20)30477-4 (2021).

15. Wilham, J. M. et al. Rapid end-point quantitation of prion seeding activity with sensitivity comparable to bioassays. PLoS Pathog 6, e1001217, doi:10.1371/journal.ppat.1001217 (2010).

16. Takatsuki, H. et al. Rapid and Quantitative Assay of Amyloid-Seeding Activity in Human Brains Affected with Prion Diseases. PLoS One 10, e0126930, doi:10.1371/journal.pone.0126930 (2015).

17. Candelise, N. et al. Applications of the real-time quaking-induced conversion assay in diagnosis, prion strain-typing, drug prescreening and other amyloidopathies. Expert Rev Mol Diagn 17, 897-904, doi:10.1080/14737159.2017.1368389 (2017). 
18. Saborio, G. P., Permanne, B. \& Soto, C. Sensitive detection of pathological prion protein by cyclic amplification of protein misfolding. Nature 411, 810-813, doi:10.1038/35081095 (2001).

19. Massignan, T. et al. A cationic tetrapyrrole inhibits toxic activities of the cellular prion protein. Sci Rep 6, 23180, doi:10.1038/srep23180 (2016).

20. Race, R., Raines, A., Raymond, G. J., Caughey, B. \& Chesebro, B. Long-term subclinical carrier state precedes scrapie replication and adaptation in a resistant species: analogies to bovine spongiform encephalopathy and variant Creutzfeldt-Jakob disease in humans. J Virol 75, 10106-10112, doi:10.1128/JVI.75.21.10106-10112.2001 (2001).

21. Gill, O. N. et al. Prevalent abnormal prion protein in human appendixes after bovine spongiform encephalopathy epizootic: large scale survey. BMJ 347, f5675, doi:10.1136/bmj.f5675 (2013).

22. Raymond, G. J. et al. Inhibition of protease-resistant prion protein formation in a transformed deer cell line infected with chronic wasting disease. J Virol 80, 596-604, doi:10.1128/JVI.80.2.596-604.2006 (2006).

23. Caughey, B., Brown, K., Raymond, G. J., Katzenstein, G. E. \& Thresher, W. Binding of the protease-sensitive form of PrP (prion protein) to sulfated glycosaminoglycan and congo red [corrected]. J Virol 68, 2135-2141, doi:10.1128/JVI.68.4.21352141.1994 (1994).

24. Imamura, M. et al. Heparan Sulfate and Heparin Promote Faithful Prion Replication in Vitro by Binding to Normal and Abnormal Prion Proteins in Protein Misfolding Cyclic Amplification. J Biol Chem 291, 26478-26486, doi:10.1074/jbc.M116.745851 (2016).

25. Shyng, S. L., Lehmann, S., Moulder, K. L. \& Harris, D. A. Sulfated glycans stimulate endocytosis of the cellular isoform of the prion protein, PrPC, in cultured cells. J Biol Chem 270, 30221-30229, doi:10.1074/jbc.270.50.30221 (1995).

26. Taubner, L. M., Bienkiewicz, E. A., Copie, V. \& Caughey, B. Structure of the flexible amino-terminal domain of prion protein bound to a sulfated glycan. J Mol Biol 395, 475-490, doi:10.1016/j.jmb.2009.10.075 (2010).

27. Teruya, K. et al. Heparinase I-specific disaccharide unit of heparin is a key structure but insufficient for exerting anti-prion activity in prion-infected cells. Biochem Biophys Res Commun 460, 989-995, doi:10.1016/j.bbrc.2015.03.139 (2015).

28. Petrosyan, R., Patra, S., Rezajooei, N., Garen, C. R. \& Woodside, M. T. Unfolded and intermediate states of PrP play a key role in the mechanism of action of an antiprion chaperone. Proc Natl Acad Sci U S A 118, doi:10.1073/pnas.2010213118 (2021).

29. Ishibashi, D. et al. Strain-Dependent Effect of Macroautophagy on Abnormally Folded Prion Protein Degradation in Infected Neuronal Cells. PLoS One 10, e0137958, doi:10.1371/journal.pone.0137958 (2015).

30. Tsuboi, Y., Doh-Ura, K. \& Yamada, T. Continuous intraventricular infusion of pentosan polysulfate: clinical trial against prion diseases. Neuropathology 29, 632-636, doi:10.1111/j.1440-1789.2009.01058.x (2009).

31. Terada, T. et al. Less protease-resistant PrP in a patient with sporadic CJD treated with intraventricular pentosan polysulphate. Acta Neurol Scand 121, 127-130, doi:10.1111/j.1600-0404.2009.01272.x (2010).

32. Kocisko, D. A. et al. New inhibitors of scrapie-associated prion protein formation in a library of 2000 drugs and natural products. J Virol 77, 10288-10294, doi:10.1128/jvi.77.19.10288-10294.2003 (2003).

33. Kocisko, D. A. et al. Comparison of protease-resistant prion protein inhibitors in cell cultures infected with two strains of mouse and sheep scrapie. Neurosci Lett 388, 106-111, doi:10.1016/j.neulet.2005.06.053 (2005).

34. Giles, K. et al. Different 2-Aminothiazole Therapeutics Produce Distinct Patterns of Scrapie Prion Neuropathology in Mouse Brains. J Pharmacol Exp Ther 355, 2-12, doi:10.1124/jpet.115.224659 (2015).

35. Teruya, K. et al. A Single Subcutaneous Injection of Cellulose Ethers Administered Long before Infection Confers Sustained Protection against Prion Diseases in Rodents. PLoS Pathog 12, e1006045, doi:10.1371/journal.ppat.1006045 (2016).

36. Nishida, N. et al. Successful transmission of three mouse-adapted scrapie strains to murine neuroblastoma cell lines overexpressing wild-type mouse prion protein. J Virol 74, 320-325, doi:10.1128/jvi.74.1.320-325.2000 (2000).

37. Nakagaki, T. et al. FK506 reduces abnormal prion protein through the activation of autolysosomal degradation and prolongs survival in prion-infected mice. Autophagy 9, 1386-1394, doi:10.4161/auto.25381 (2013).

38. Yamasaki, T., Suzuki, A., Hasebe, R. \& Horiuchi, M. Comparison of the anti-prion mechanism of four different anti-prion compounds, anti-PrP monoclonal antibody 44B1, pentosan polysulfate, chlorpromazine, and U18666A, in prion-infected mouse neuroblastoma cells. PLoS One 9, e106516, doi:10.1371/journal.pone.0106516 (2014). 
39. Hayashi, H. et al. Effect of tissue deterioration on postmortem BSE diagnosis by immunobiochemical detection of an abnormal isoform of prion protein. J Vet Med Sci 66, 515-520, doi:10.1292/jvms.66.515 (2004).

40. Fujihara, A. et al. Hyperefficient PrP Sc amplification of mouse-adapted BSE and scrapie strain by protein misfolding cyclic amplification technique. FEBS J 276, 2841-2848, doi:10.1111/j.1742-4658.2009.07007.x (2009).

41. Yamasaki, T. et al. Characterization of intracellular localization of $\operatorname{PrP}(\mathrm{Sc})$ in prion-infected cells using a mAb that recognizes the region consisting of aa 119-127 of mouse PrP. J Gen Virol 93, 668-680, doi:10.1099/vir.0.037101-0 (2012).

\section{Figures}

A

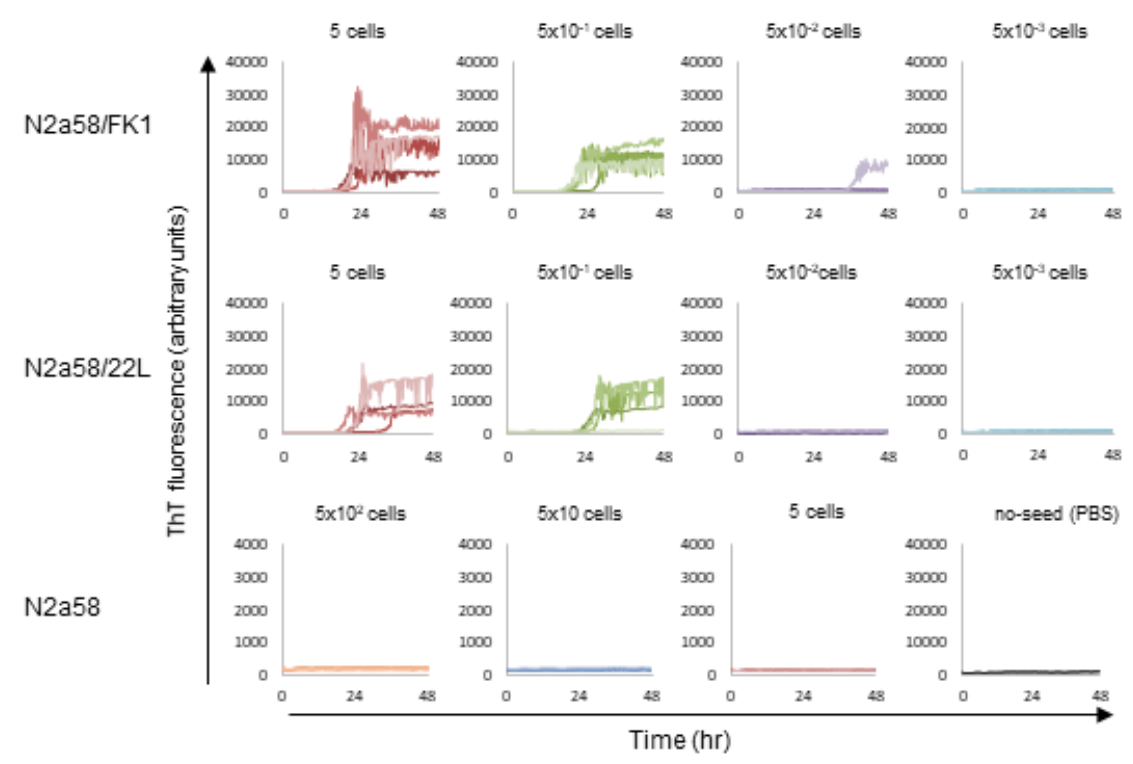

B
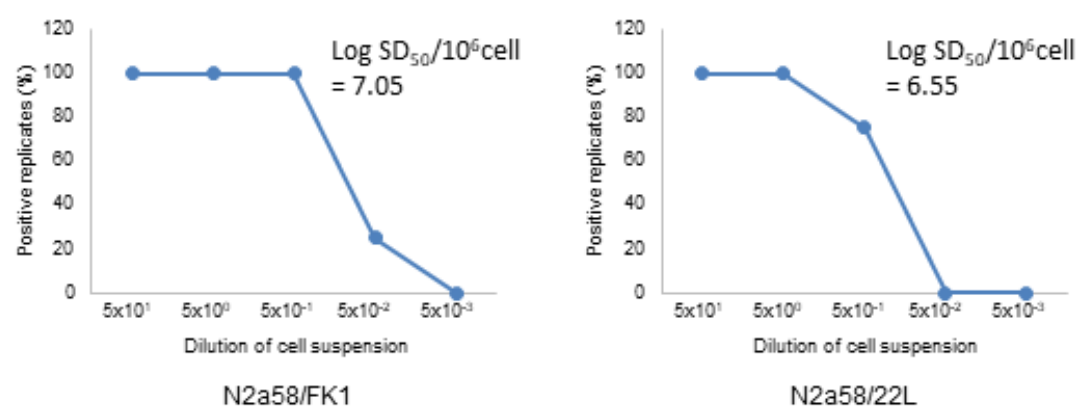

Figure 1

Quantification of seed activity in prion-infected cells using Endpoint RT-QuIC.

(A) RT-QulC assay of serially diluted prion-infected cell lysates ( $n=4$ for each group). (B) Positive reaction rate at each dilution. A positive result was obtained when the fluorescence value was more than twice the base value. 

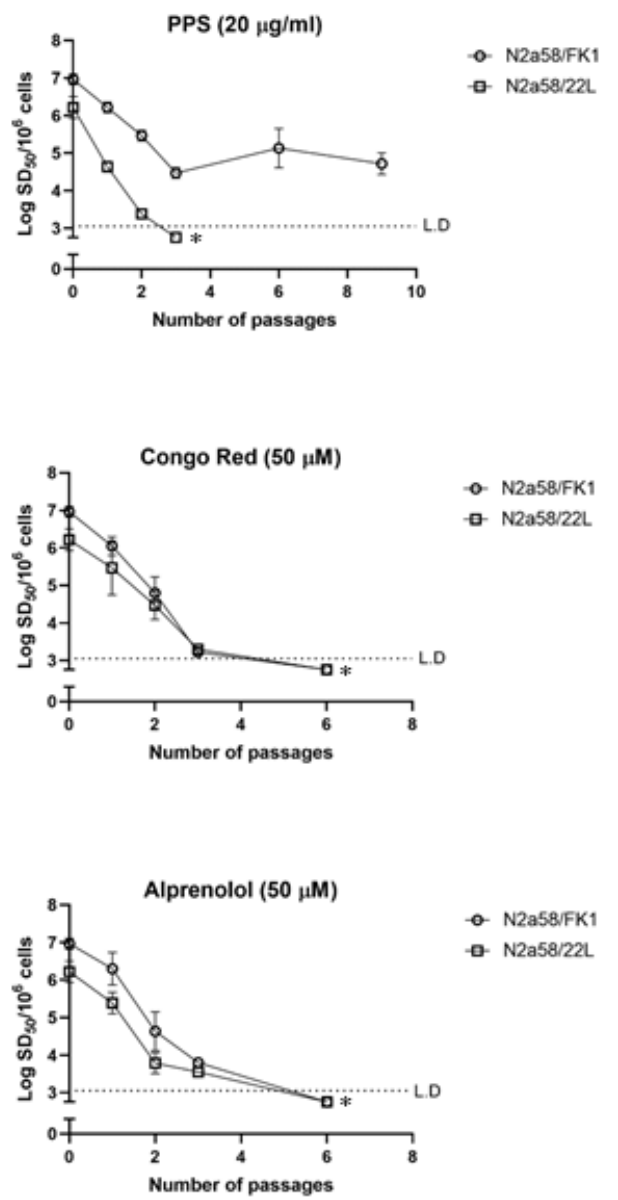

\section{Figure 2}

\section{Seeding activity in prion-infected cells treated with anti-prion compounds.}

Prion-infected N2a58 cells were treated with PPS, Congo red, or alprenolol from P0-P6. For each passage, prion seeding activity ( $\log \mathrm{SD}_{50} / 10^{6}$ cells) was quantified using RT-QuIC $(\mathrm{n}=3)$. Open circles and open squares indicate N2a58/FK1 and N2a58/22, respectively. Data represent mean \pm standard deviation (SD). Asterisk indicates below detection limit. 


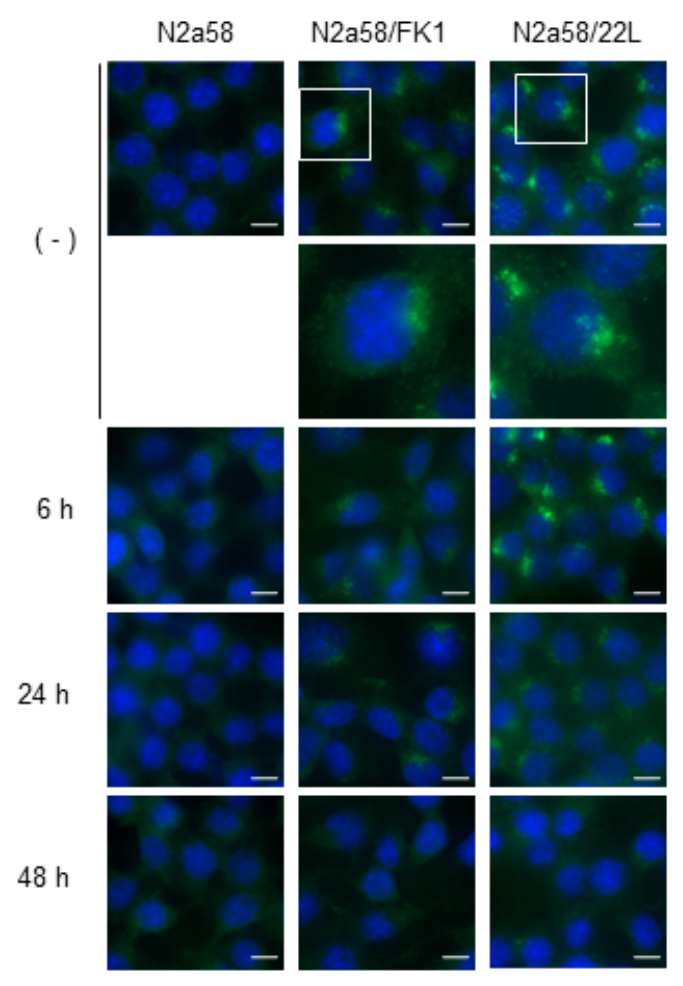

Figure 3

Influence of PPS on intracellular PrPSc.

Prion-infected N2a58 cells were cultured with $20 \mathrm{mg} / \mathrm{ml}$ of PPS for 6,24 , or $48 \mathrm{~h}$. (-) indicates no PPS treatment. Panels show merge

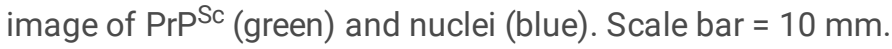



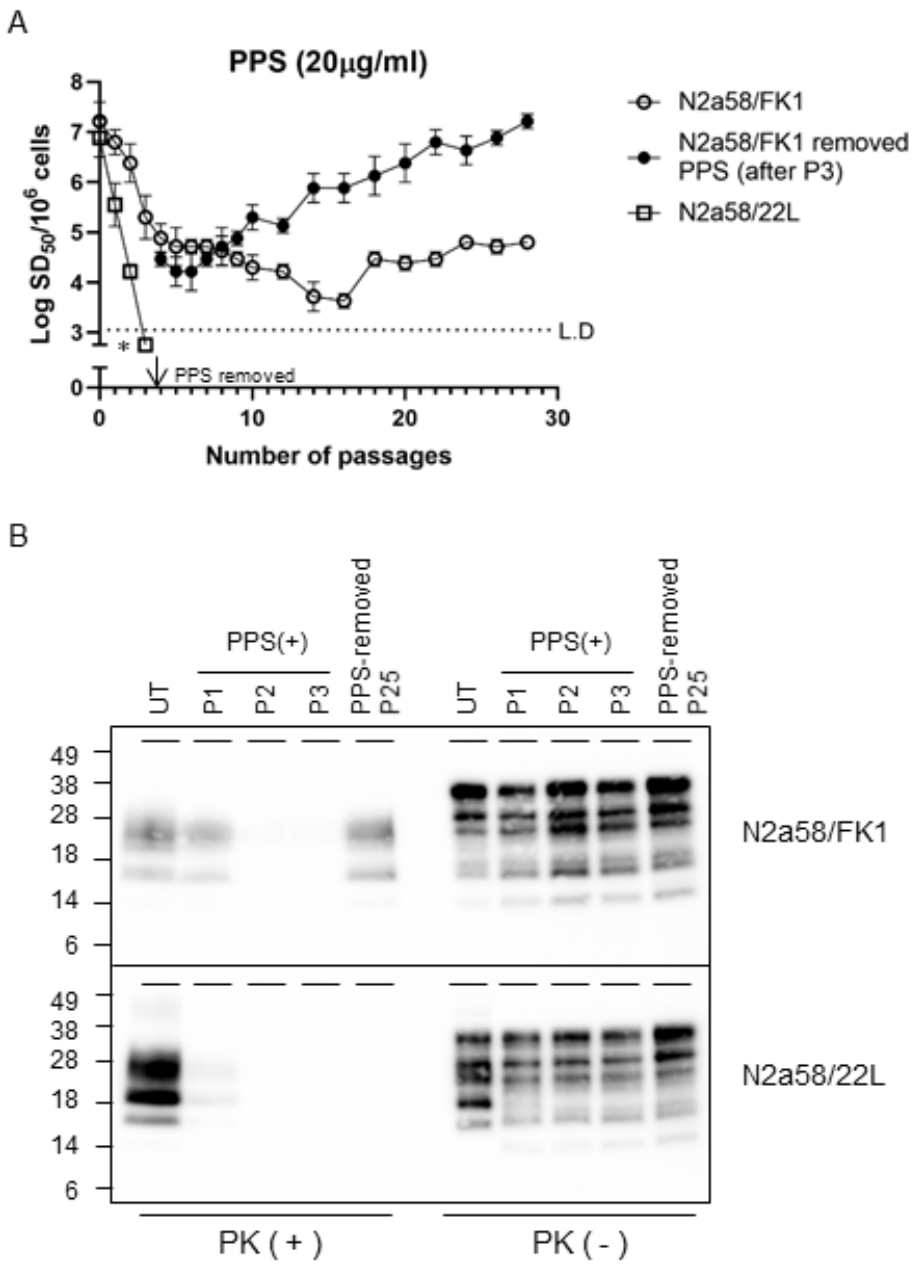

Figure 4

Timecourse of seeding activity and PrP-res levels of prion-infected cells treated with PPS continuously or removed after P3.

(A) Open circles and open squares indicate N2a58/FK1 with PPS and N2a58/22L with PPS, respectively. Closed circles indicate N2a58/FK1 cultured in medium without PPS after P3. Data represent mean \pm SD. Asterisk indicates below detection limit. (B) PrP-res $[\mathrm{PK}(+)]$ and total PrP [PK(-)] were analyzed by WB in P1-P3 prion-infected cells cultured in the presence of PPS and P25 prioninfected cells after excluding PPS. Total protein content of N2a58/FK1 cell lysate per lane was $60 \mathrm{mg}$ and $20 \mathrm{mg}$ for PK(+) and PK(-), respectively; for N2a58/22L, $45 \mathrm{mg}$ and $15 \mathrm{mg}$ were used for PK(+) and PK(-), respectively. UT represents untreated cells. The full-length blot image is provided in the Supplementary Figure 1. 
A
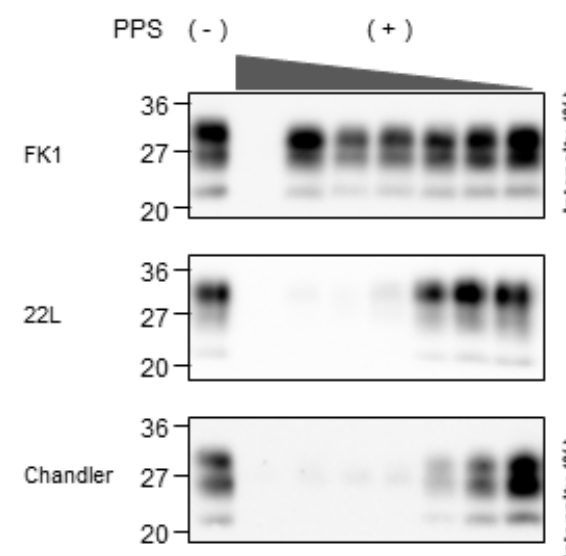

ME7

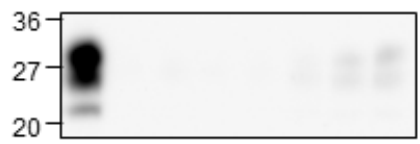

mBSE

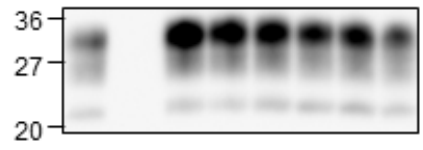

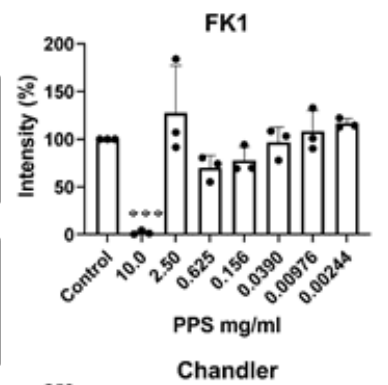
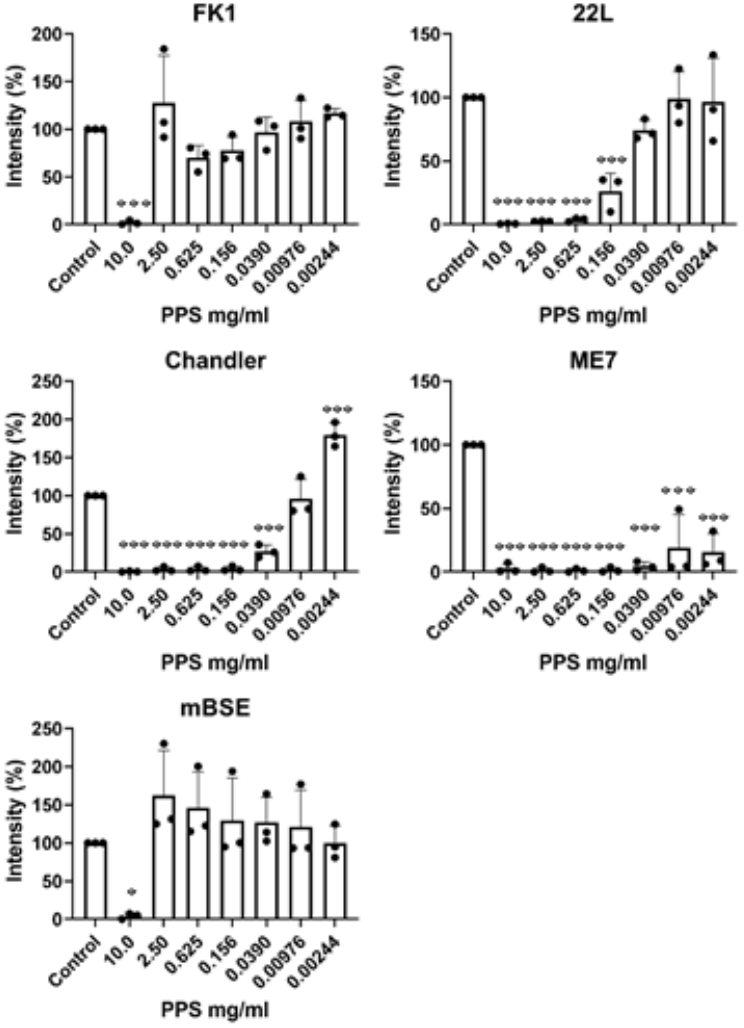

Figure 5

\section{Inhibitory effect of PPS on PMCA.}

(A) Brain homogenates of prion-infected mice were used as seeds for PMCA with a four-fold serial dilution of PPS (10 mg/ml, 2.5 $\mathrm{mg} / \mathrm{ml}, 0.625 \mathrm{mg} / \mathrm{ml}, 0.156 \mathrm{mg} / \mathrm{ml}, 39 \mathrm{mg} / \mathrm{ml}, 9.8 \mathrm{mg} / \mathrm{ml}$, and $2.4 \mathrm{mg} / \mathrm{ml}$ ). Representative images of WB of PrP-res are shown. The corresponding full-length blot images, and blots for each of the three independent PMCA experiments are provided in the Supplementary Figures 2-5. (B) Mean \pm SD of band intensities are shown in the bar graph ( $\mathrm{n}=3$ for each group). Control represents PMCA without PPS and is set at $100 \%$. Statistical analysis was performed using one-way ANOVA followed by Dunnett's test. *P < $0.05, * \star p<0.01, * \star * p<0.001$ compared with control.

\section{Supplementary Files}

This is a list of supplementary files associated with this preprint. Click to download.

- Supplementarylnformationed5.pdf 